\title{
EVALUATION OF DIATHERMY
}

\section{$B Y$ DISRAELI KOBAK, M.D.}

This interesting paper never appeared in a Medical Journal but was printed as a private publication after Kobak' death by Cecil Birtcher, President of the Birtcher Corporation, who was kind enough to permit us to reprint it.

\begin{abstract}
N September, 1955, 1 had dinner at the Blackstone Hotel in Chicago with Dr. Disraeli Kobak. Dr. Kobak handed me I a paper which he stated he intended to present before a medical group in the near future. As it must to all men, death came to Dr. Kobak a few short months later and his paper was never presented.
\end{abstract}

As editor for seventeen years of the Magazine, Archives of Physical Medicine, X-ray and Radium, the official journal of the American Congress of Physical Medicine, the wise, kindly, thought-provoking, and sometimes vitriolic words of Dr. Kobak were read throughout the world. It is therefore in deep respect and memory of Dr. Disraeli Kobak that perhaps what is his last paper is now privately published that his good words may again bring thoughts to the many, many thousands of physicians and therapists throughout the world who were his friends and followers.

\section{Cecil Birtcher, President, The Birtcher Corporation.}

It has been reported to me that recently an eminent physician while addressing a sizeable group of registered Physical Therapists on the many aspects of physical medicine, passed lightly over the subject of diathermy with the remark, "Diathermy never cured anything".

Such a remark might well have been directed to any method within physical medicine or for that matter to the million and one drugs in the Pharmacopea. Surgery can remove a diseased organ and the patient recover sufficiently to seem to enjoy a full measure of health but the diseased organ was not "cured" nor is the patient a whole man.

Such a remark from a speaker's platform to the tyro in physical medicine is unfortunate. The speaker was damned with faint praise. Such a remark discourages the use of a method which has proved itself for over forty years in the hands of over 200,000 physicians throughout the entire world.

In spite of the shortcomings of diathermy and of the many who employ it there is no one method in physical medicine, nor among all the drugs so reliable or so broadly indicated in daily use of a physician than diathermy. No, the often startling results achieved by our modern miracle drugs have not in any way tarnished the brilliant achievements of diathermy so long established, doing its daily perhaps unspectacular job a million times each day.

Because diathermy has become so familiar in the daily practice of the physician and the trained physical therapist it does not follow that the old adage "Familiarity breeds contempt" should mire the scientific thinking and practice of those trained in physical medicine.

The fact that the early enthusiasms for the vitamins, the antihistamines, the modern hormone drugs and the amazing antibiotics have now been most modestly and conservatively evaluated does not refute their respective importance nor should it discourage the continued use of these drugs by the medically trained.

The fact that it has been established that certain virus have developed strains resistant to antibiotics even in patients never previously treated with these drugs does not suggest that the antibiotics be discarded in practice nor in further research.

By the same token diathermy must not, and cannot be discounted by the thinking earnest physician. Diathermy is familiar and commonplace but much as yet must be learned about it. There is a great opportunity for research as well as clinical usage.

A diathermy machine introduces high frequency electrical energy into tissue. When a sufficient amount of energy is introduced it manifests itself by the dissipation of the energy in the form of heat deep in the underlying tissues. Temperatures are regularly created within the tissue at $102^{\circ} \mathrm{F}, 105^{\circ}$, $106^{\circ}$ or more, or less as directed by the operator. It has been established that this heat dilates the arterial, venous and capilliary passages and materially increases the flow of

blood through the heated part. It has been reasoned therefore that all clinical value of diathermy is due to whatever value exists due to the materially increased blood circulation in the treated part. That in itself is good but it does leave many questions unanswered.

Heat is generated in tissue when the excess energy must be dissipated. How about the energy that is introduced into the tissue that is at a level less than heat producing? There are many sincere and competent medical men who have regularly observed excellent clinical results employing high frequency energy from a diathermy machine at such low levels of energy that there has been no measurable temperature rise in the tissue whatsoever. Why does diathermy at a high level of energy and heat production aggravate some painful symptoms and alleviate other painful symptoms? Diathermy at a high level of energy seems to materially increase the pain in neuritis and yet at a very low level very often seems to give amazing relief to the patient.

What dosage do we give to a patient with diathermy? How many watts of electrical energy do we introduce into tissue? As of this writing there is no diathermy machine which even indicates the actual amount of energy being delivered into a patient. Meters on diathermy machines only indicate a relative increase of energy output but even this with no degree of accuracy in terms of watts, the electrical unit of measurement.

What chemical changes take place in tissue with a diathermy treatment? If any, is this the effect of the heat produced or due to some activation created by the flow of the electrical energy? This question is still a controversial issue in view of the inadequacy of accurate measurement of energy delivered into tissue and because of the diverse opinions by equally distinguished medical investigators.

The broad use of diathermy does not contraindicate the use of drugs or other physical measures. In fact unless making a special controlled study all other methods should be employed with diathermy each an adjunct to the other. Let us see what some of the most conservative publications have recently said about diathermy. The Council on Physical Medicine of the American Medical Association recommends the use of diathermy on the following diseases very often in conjunction with other therapy, drugs, or sometimes alone.

Contusions

Muscle Sprains

Myositis Ossifications

Sprains and Dislocations

Bursitis

Tenosynovitis

Chronic Arthritis

Myositis and Myofascitis Fibrositis

The January 7, 1950 issue of the Journal of the A.M.A.

carried a comprehensive article on medical diathermy by

Fractures

Genito-Urinary Conditions

Pelvic Infections

Respiratory Diseases

Gastrointestinal Diseases

Inflammation of the

Peripheral Nerves

Acute and Chronic Sinusitis

Eye Diseases
Suppurative Processes 
Gordon M. Martin, M.D. and Donald J. Erickson, M.D. of the Section of Physical Medicine of the Mayo Clinic and have reported their usage of medical diathermy on the following:

Rheumatoid Arthritis

Osteo-arthritis or Hypertrophic Arthritis

Bursitis

Tenosynovitis

Fibrositis and Myositis

Sprains and strains

Fractures

Neuritis

\section{Peripheral Vascular \\ Disease}

\section{Sinusitis}

Otitis Media

Pelvic Inflammatory Disease

Pleurisy

Diverticulitis

Infection of the eye

Now that such a larger percentage of our population is elderly and this is increasing regularly the use of diathermy for older people is of increasing importance. The August, 1948 issue of The Medical Record carried an article entitled "Value of Physical Therapy as an "Adjunct for the Aged" by Louis Feldman, M.D. of Brighton, Massachusetts. Dr. Feldman reports diathermy of value in these elderly folk in the treatment of varicose veins, chronic cholecystitis, gastric neurosis, atonic dyspepsia, benign prostatic enlargement and chronic prostatitis, spasm of the ureter with urethral stone or gout, osteo and rheumatoid arthritis, fibrositis, myositis, bursitis, stiff joints and for lessening muscular rigidity and improvement of motion in paralysis agitans.

The November, 1949 issue of Medical Economics carried the article "Diathermy Equipment for the G.P." by Edwin Matlin, M.D., Dr. Matlin reported that his practice is located in a village of approximately 1,800 inhabitants in Pennsylvania. He reported that in diathermy he was giving a tangible service to his patients. Though by no means does he specialize in physical medicine he reported that during the six months' period immediately preceding he had seen an average of 15 patients per day. During the six months the cases treated by diathermy were as follows:

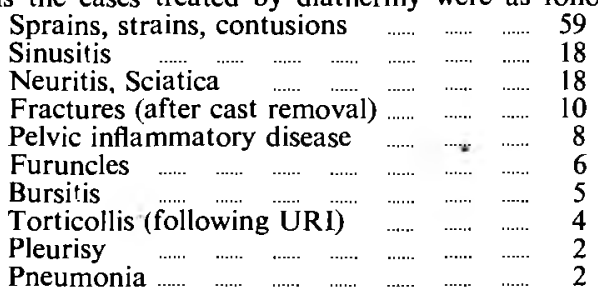

Yes, diathermy conservatively evaluated has an extremely broad usage. Perhaps in many cases a drug will do as well. A bit of codine or five or six ounces of whiskey will probably relieve a dysmenorrhea, but is it desirable to introduce a very toxic drug into this patient which may become habit forming?

To go back to my first paragraph and the good doctor who carelessly said, "Diathermy never cured anything", I shall agree with him but I object to his failure to make the remark without further comment. No, Doctor, diathermy never cured any'hing, but perhaps you hair has not grown grey with mine in usage of diathermy for over 30 years. Perhaps you have never given six one hour diathermy treatments in a 24 hour period to an apparently hopeless lobar pneumonia as I have and on many occasions seen the temperature declined by lysis. Perhaps you have never treated an agonized acute lumbago four one hour treatments in a single day for two days then observed that patient on a tennis court the fourth day. I have.

Now, diathermy never cured anything but I am mighty glad this wonderful tool of medicine is available to my hands in my daily practice.

Let us take the familiar, the commonplace, the reliable method of 50 years and guide it with intelligence and with the sincerity, enthusiasm and energy of a missionary, not to forego interest and the stimulation of the new but to revitalize and renew the old.

\section{This hygienic}

\section{Physiotherapist Uniform has so many good points!}
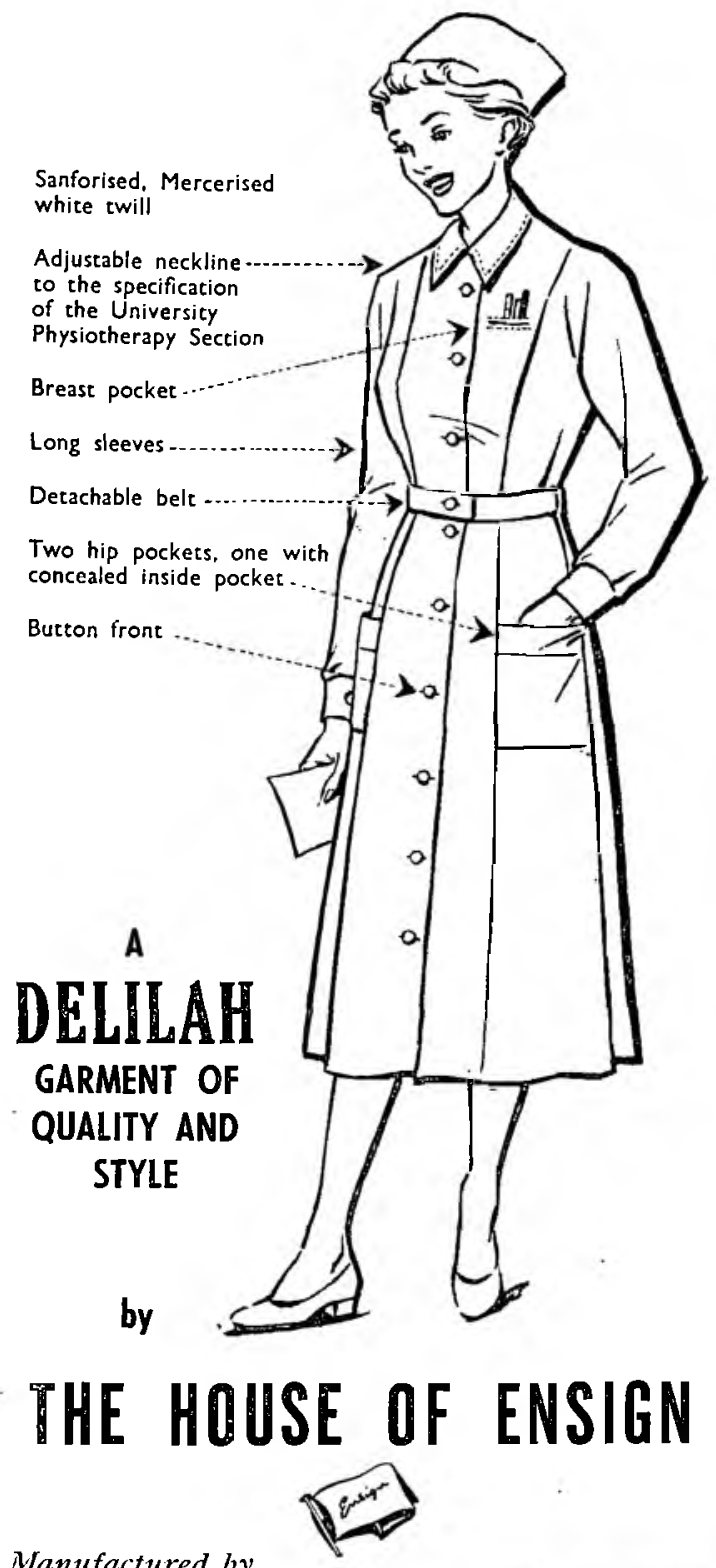

Manufactured by

The AFRICAN CLOTHING FACTORY (Susign) Ltd.

P.O. BOX 1098 CAPE TOWN

and obtainable from good clothing stores everywhere 